\title{
Detection of stress functional responses in bacterial populations under dry soil conditions show potential microbial mechanisms to resist drought conditions
}

\author{
Soumyadev Sarkar ${ }^{1}$, Kaitlyn Ward ${ }^{1}$, Janet K. Jansson ${ }^{2}$, Sonny T.M. Lee ${ }^{1 *}$ \\ ${ }^{1}$ Division of Biology, Kansas State University, Manhattan, Kansas, United States \\ ${ }^{2}$ Earth and Biological Sciences Directorate, Pacific Northwest National Laboratory, Richland, \\ Washington, United States \\ *Corresponding author, E-mail: leet1@ksu.edu
}

\begin{abstract}
Climate change is predicted to have a negative effect on the grasslands of the United States and will be detrimental to the economy and environment. The changing precipitation levels would also have an effect on the structural and functional potential of associated soil microbiome communities, which in turn will regulate the health of the plants during stressful conditions. In this study, we applied metagenomics analyses to capture the responses of the bacterial populations under drier soil conditions. We collected soil from two sites (dry and wet) at the Konza LongTerm Ecological Research field station in Kansas, which had characteristic features of the native prairies. Soil drying resulted in a significant shift in the bacterial population at the community level. Following that, fifteen bacterial genomes were short-listed based on the availability in the public database, higher relative abundance in dry soils than in wet, and also according to their contributions in drier soil. The potential microbial mechanisms were elucidated when an in-depth analysis of the functional genes was performed. Translation elongation factor EF-Tu, thiamine biosynthesis protein, and catalase were identified as a part of the overall stress functional responses in the bacterial population in this study. We speculate that these identified bacterial populations are important for maintaining the health of the soil under dry conditions. Genes and/or pathways found in this study provide insights into microbial mechanisms that these bacterial populations might employ to resist challenging drought conditions.
\end{abstract}

Keywords: metagenome, dry soil, drought, soil microbiome 


\section{Introduction}

Climate change is an incessant global concern that often results in altered precipitation levels, seasonal fluctuations, warming ocean floors, etc. (VijayaVenkataRaman, Iniyan, and Goic 2012). Events like altered rainfall have a direct negative effect on agriculture and productivity (Gornall et al. 2010). In the United States, future irregularity in rainfall is predicted in the south-central, south-western, and in the northern and central grasslands of North America (Cook, Seager, and Miller 2011; Intergovernmental Panel on Climate Change 2014; Taylor et al. 2012). The increased rainfall irregularity in the mesic grasslands is associated with a reduction in soil respiration (Schimel 2018), reduced aboveground net primary productivity (ANPP) (Knapp et al. 2002; Heisler-White et al. 2009), decrease in carbon cycling processes (Heisler-White et al. 2009), altered nitrogen cycling processes (Schimel 2018), and even in decline in the level of photosynthesis (Jones et al. 2016). There are also reports of change in genotypic diversity in the grasslands as a consequence of rainfall alterations (Jones et al. 2016; Avolio, Beaulieu, and Smith 2013).

These modifications happen not only in the host but also in the associated microbiome as well (Chowdhury et al. 2019). Under changing precipitation profiles, microbiomes exhibit osmotic adjustments, optimum resource efficiency, enhanced microenvironment, stable dormancy to evade stress (Schimel 2018). It is speculated that the plant host and -associated microbiome remain complementary towards each other in this changing scenario (Lau and Lennon 2012). The shift in the microbiome has a definite impact on the plant fitness under dry conditions, and have contributed towards increasing the health of the plants during drought $(\mathrm{Xu}$ and Coleman-Derr 2019).

The cellular and functional responses by bacteria populations during drought conditions include induction of dormancy (forming spores) (Barnard, Osborne, and Firestone 2013), production of exopolysaccharides (Roberson and Firestone 1992), performing osmotic adjustments (Chowdhury et al. 2019), synthesis of enzymes that are extracellular in nature to sequester solutes (Chowdhury et al. 2019; Barnard, Osborne, and Firestone 2013). During drought conditions, Actinobacteria and Planctomycetes use pathways related to osmotic stress responses (Bouskill et al. 2016). Exopolysaccharides are produced in Pseudomonas sp. and Acidobacteria to 
resist desiccation (Roberson and Firestone 1992; Chang et al. 2007; Ward et al. 2009). Bacillus spp. and Actinobacteria possess the ability to form spores in desert-like conditions (Marasco et al. 2012).

Until now, investigations to understand soil microbiome processes under dry conditions have focussed on a more holistic approach to identifying diverse microbial populations without extracting much information about the underlying mechanisms. Although there are studies that attempt to identify the shifts in soil microbial functions under stressful conditions, there is still a clear knowledge gap that exists on how these mechanisms can be associated with the participating bacterial population during drought (Riah-Anglet et al. 2015; D. Liu et al. 2019). This study aims to fill this void, and we used metagenomic approaches at the (a) community level (b) genome level (c) functional genes level to link the impact of soil drying with mechanisms that the microbial communities adopt to resist such extreme conditions.

We collected soil samples with distinct moisture characteristics (wet and dry) from two locations in Konza Long-Term Ecological Research field station (Kansas), to investigate how changes in soil moisture levels would induce a shift in the bacterial population and in the participating metabolic genes and pathways. Our study yielded detailed information on the bacterial community, genome, and gene level, and we believe this lays the framework for other similar scientific explorations.

\section{Materials and Methods}

\subsection{Sample sites and collection}

In this study, soils were collected from two sites in Konza Long-Term Ecological Research field station in Kansas. Soil from site A $\left(39^{\circ} 06=11\right.$ N, $96^{\circ} 36=48$ W, 339 meters above sea level) had a higher water content of $37 \%$, whereas site $\mathrm{B}\left(39^{\circ} 04=39 \mathrm{~N}, 96^{\circ} 36=29 \mathrm{~W}, 413\right.$ meters above sea level) soil had a lower water content of $18 \%$. Both the sampling locations had $\mathrm{C} 4$ perennial grasses, resembling the characteristics of native prairies, and were not disturbed by agricultural practices.

Soil samples $(\mathrm{n}=12)$ were collected at a depth of $15 \mathrm{~cm}$ from three locations which were minimum $10 \mathrm{~m}$ apart, from both sites. After removing rocks and roots, the soils were homogenized and then collected in single Ziploc bags for each site. Soils were immediately frozen in liquid nitrogen, and shipped. After receiving at the Pacific Northwest National Laboratory (PNNL), 
Richland, WA, the soil samples were sieved, aliquoted in $50 \mathrm{ml}$ conical centrifuge tubes and stored at $-80^{\circ} \mathrm{C}$ until further processing.

\subsection{Shotgun metagenomics and reference-based metagenomics}

DNA extraction was carried out as previously described (Chowdhury et al. 2019). Sequencing was performed on the HiSeq 2500 platform (Illumina, San Diego, CA) which generated 250-bp pairedend reads $(\sim 10$ million reads per sample) from three runs. We used Kaiju ver 1.7.2 (Menzel, Ng, and Krogh 2016) to perform taxonomic classification of the high-throughput reads from the metagenomes. Clustering algorithms were executed with a 99\% similarity cut off, and each sequencing read was assigned a taxon against the RefSeq database. Chimeras and sequences corresponding to plants/vertebrates were also filtered.

We short-listed and downloaded 15 genomes ( $\sim 5$ million bp) from the National Center for Biotechnology Information database (Sayers et al. 2020) (Supplementary Table S1). We chose these fifteen bacterial genomes based on the following criteria: 1) the bacterial populations' higher mean relative abundance in the dry soil indicated by Kaiju analysis at the species level 2) based on their availability of the genomes in the database, and 3) contribution to the dissimilarity as analyzed from SIMPER analysis of the whole community at the species level. We downloaded the following genomes - Azosprillum brasilense, Azospirillum thiophilum, Azotobacter chroococcum, Azotobacter vinelandii, Candidatus Nitrospira inopinata, Candidatus Nitrospira defluvii, Thiobacillus denitrificans, Nitrosococcus halophilus, Nitrosococcus watsonii, Nitrosococcus oceani, Paracoccus denitrificans, Nitrospira moscoviensis, Frankia casuarinae, Frankia inefficax, and Frankia Datisca glomerata.

We mapped short-reads from each of the 12 metagenomes onto the 15 downloaded bacterial genomes using Bowtie2 ver 2.3.5.1 (Langmead and Salzberg 2012), and stored the recruited reads using samtools (Li et al. 2009). We then used anvi'o ver 6.1 (Eren et al. 2015) to process the BAM files and generate profile databases containing coverage and detection statistics of each downloaded genomes in the metagenomes.

\subsection{Statistical analysis}

We used primer v7 (Clarke and Gorley 2015) to perform all statistical analyses. For community and genome analyses, we standardized and square-root transformed (Christian, Kaestli, and Gibb 
2017) the data, and tested for differences in the bacterial community using PERMANOVA analysis on Bray-Curtis similarity. SIMPER statistical analysis (cut-off: 70\%) was also performed during community, genome, and functional genes analyses. We used ggplot 2 in the R tidyverse package to visualize and create all figures (Gómez-Rubio 2017).

\section{Results and discussions}

To understand the soil bacterial membership and function between two soil types with distinct precipitation levels, we sampled two locations that had a defined soil moisture content difference (soil A: 37\% and soil B: 18\%) (Chowdhury et al. 2019). We categorized soil A as wet soil, and soil B as dry throughout this report. Our study added to the current knowledge on soil microbial functional shifts during stressful conditions. In this study, we have focused on the functional contributions, and our findings are based not only on the basis of the relative abundance but also on contributions of each member(s) at each level, i.e. community, genomes and genes. Our findings support that there is a distinct community shift upon soil drying which is consistent with previous studies (Zhou et al. 2016; Pajares et al. 2018). Besides the confirmation that there are clear impacts on the community as a whole, we also noticed that new members of bacterial populations being more abundant in both conditions (dry and wet), which were not previously reported in a single study and/or similar soil profile(s). Our study thus provided the baseline that enabled each of the distinct bacterial populations to be studied further to enrich our current knowledge.

\subsection{Soil drying influences the microbial community structure}

Soil A generated $\sim 64$ million reads and soil B generated $\sim 71$ million reads. We assembled the reads with a minimum contig length of $1000 \mathrm{bp}$, yielding $\sim 47,000$ and 51,000 contigs in soil A and B respectively (Supplementary Table S2). We used Kaiju ver 1.7.2 (Menzel, Ng, and Krogh 2016) to elucidate the impact of soil moisture content on the soil microbial community. Briefly, each of the reads in the metagenomes is assigned to a taxon in the NCBI BLAST non-redundant protein database. The reads are translated into amino acid sequences, and are searched in the database. We classified 365 families in both the soil locations (Supplementary Table S3). We 
showed a statistical difference (PERMANOVA, Pseudo-F 35.643, P < 0.05, Figure 1) in microbial community structure between the two distinct soil types (dry and wet).

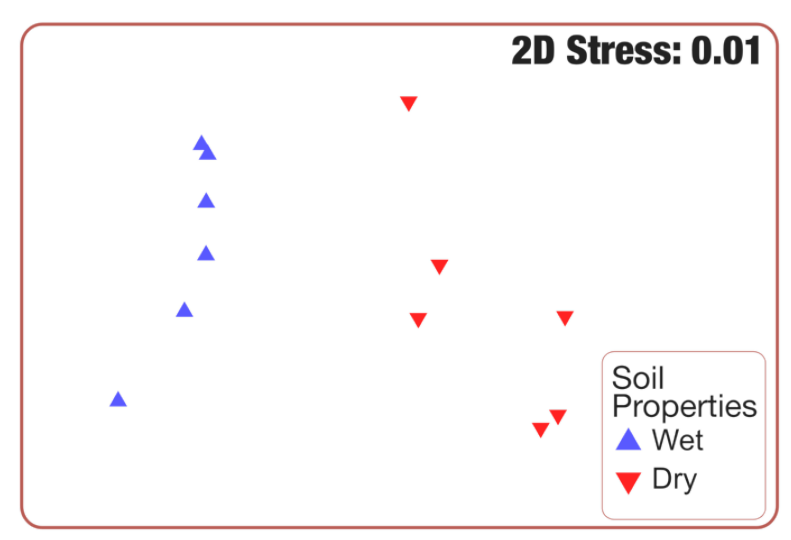

Figure 1

We noticed, through post-hoc SIMPER analyses, that Streptomycetaceae (1.21\%), Bradyrhizobiaceae (1.2\%), Vicinamibacteraceae (1.13\%), Pseudonocardiaceae (0.97\%), Planctomycetaceae (0.9\%) contributed the most in dry soil (Figure 2A, Supplementary Table S4). Following that, Sphingomonadaceae, Burkholderiaceae, Hyphomicrobiaceae, Comamonadaceae, Conexibacteraceae, Mycobacteriaceae, Micromonosporaceae, Acidobacteriaceae, Rhizobiaceae, Rhodospirillaceae, Chitinophagaceae, Polyangiaceae, Methylobacteriaceae, Solibacteraceae, and Phyllobacteriaceae also were among the top contributors. In the wet soil, Bradyrhizobiaceae (1.57\%), Streptomycetaceae (1.21\%), Hyphomicrobiaceae (0.99\%), Pseudonocardiaceae $(0.97 \%)$, and Vicinamibacteraceae were the top contributors (Figure 2B, Supplementary Table S5). Micromonosporaceae, Chitinophagaceae, Acidobacteriaceae, Mycobacteriaceae, Burkholderiaceae, Conexibacteraceae, Sphingomonadaceae, Planctomycetaceae, Comamonadaceae, Solibacteraceae, Polyangiaceae, Nocardioidaceae, Methylobacteriaceae, Rhizobiaceae, Gemmatimonadaceae, also contributed in wet soil. 


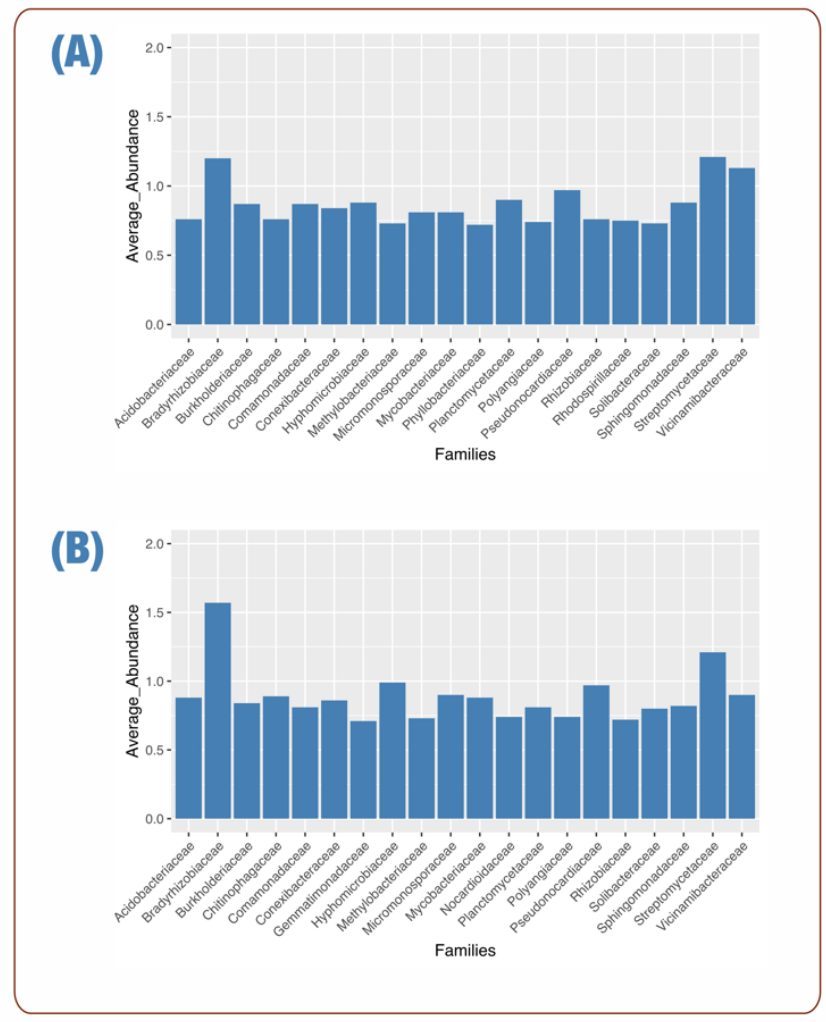

Figure 2

Vicinamibacteraceae (dry: 1.13\%, wet: 0.9\%), Sinobacteraceae (dry: 0.59\%, wet: 0.4\%), Xanthomonadaceae (dry: 0.65\%, wet: 0.52\%), Christensenellaceae (dry: 0.27\%, wet: 0.16\%), Planctomycetaceae (dry: 0.9\%, wet: 0.81\%), Rhodospirillaceae (dry: 0.75\%, wet: 0.68\%), Woeseiaceae (dry: 0.27\%, wet: 0.2\%), Comamonadaceae (dry: 0.87\%, wet: 0.81\%), Acidiferrobacteraceae (dry: $0.36 \%$, wet: $0.3 \%$ ), were the top contributors that showed statistically higher average abundance in dry as compared to wet soil (Figure 3, Supplementary Table S6). On the other hand, the wet soil as compared to dry soil, showed statistically higher average abundance of Bradyrhizobiaceae (dry: 1.2\%, wet: 1.57\%), Acidobacteriaceae (dry: 0.76\%, wet: 0.88\%), Geodermatophilaceae (dry: 0.45\%, wet: 0.57\%), Nocardioidaceae (dry: 0.63\%, wet: 0.74\%), Hyphomicrobiaceae (dry: 0.88\%, wet: 0.99\%), Micromonosporaceae (dry: 0.81\%, wet: 0.9\%), Propionibacteriaceae (dry: 0.43\%, wet: 0.51\%), Mycobacteriaceae (dry: 0.81\%, wet: 0.88\%), among others. During our analysis of the top-contributors (Supplementary Table S6), Bradyrhizobiaceae (dry: 1.2\%, wet: $1.57 \%$ ) was one of the families that had high average abundance and contribution in both the wet and the dry soil, suggesting that soil moisture had little significant impact on its presence. 


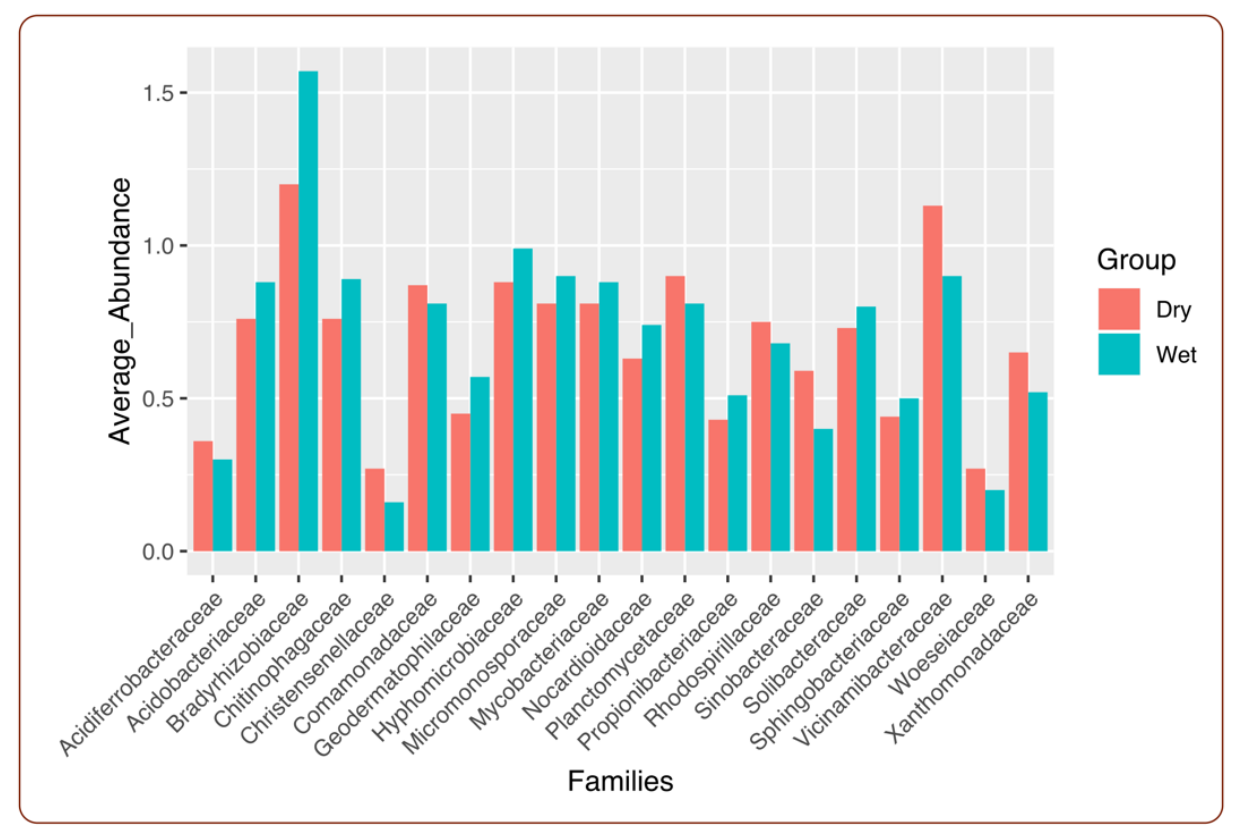

Figure 3

Our metagenome analyses inferred that the soil moisture content dictated the microbial composition. In the previous studies, dry soil induced a similar microbial population profiling (Chowdhury et al. 2019). We further discovered that Actinobacteria, Acidobacteria, Proteobacteria, Planctomycetes, Bacteroidetes were the top contributing bacterial populations in soil with a lower moisture content, and may have significant biological importance under drought conditions. For example, Actinobacterium isolated from semi-arid environments has been reported to enhance the drought tolerance in maize (Selim et al. 2019), while Acidobacteria, Proteobacteria, and Bacteroidetes are ubiquitous in soils thus explaining its importance (Chowdhury et al. 2019; Chodak et al. 2015). Acidobacteria has been reported to exist in nutrient poor soil with low carbon availability (Fierer, Bradford, and Jackson 2007). Planctomycetes, on the other hand, has some interesting features: (a) large genomes (b) lack of peptidoglycan in the cell walls, and (c) division of cells by inner membranes into separate compartments, which might be responsible for its contribution and resistance towards drought (Daniel H. Buckley et al. 2006).

SIMPER analysis indicated that there were $\sim 110$ families that were overlapping in terms of average relative abundance in both wet and dry soil (Supplementary Table S7). In order to understand the true picture of the community shift, we looked into the families that were exclusive to the dry and the wet. The bacterial families that were found to be present exclusively in the wet 
soil include Akkermansiaceae, Beijerinckiaceae, and Glycomycetaceae. Akkermansiaceae belongs to the phylum Verrumicrobia. Members of the phylum Verrumicrobia are ubiquitous in soil, and are extremely sensitive to environmental alterations, which might explain its presence in wet and not in dry soil in this study (Kielak et al. 2008; Bruce et al. 2010; D. H. Buckley and Schmidt 2001; Pan et al. 2014; Acacio Aparecido Navarrete, Diniz, et al. 2015; Acacio A. Navarrete et al. 2015; Acacio Aparecido Navarrete, Soares, et al. 2015). Beijerinckiaceae shows an ability of fixing nitrogen, even able to grow in nitrogen limiting conditions (Marín and Arahal 2014), providing essential conditions in aiding plant growth (Thuler et al. 2003; Miyasaka et al. 2003). Glycomycetaceae are soil isolates, belonging to the phylum Actinobacteria (Stackebrandt 2014).

On the other hand, we identified Alicyclobacillaceae, Christensenellaceae, Oceanospirillaceae, Rhodocyclaceae, Vulgatibacteraceae, and Woeseiaceae, which were exclusively in dry soil. Members of the Alicyclobacillaceae are capable of forming endospores and have been isolated from extreme environments, and it might explain why this family was thriving in dry soil conditions (Imperio, Viti, and Marri 2008; Goto et al. 2003). Christensenellaceae are well-known for their characteristics of being fermentative, and demonstrating the capabilities of reducing iron and sulfur, which might allow them to show its existence in such a water stressed environment (Gupta et al. 2018). Oceanospirillaceae has been previously isolated from soils contaminated with oil-sludge and oil-pollutions. So, this supports the fact of its prevalence in the drier soil here (Kuang et al. 2018; Chikere et al. 2019). Members of the Rhodocyclaceae have been isolated from a diverse range of environments i.e. soil, plants dealing with sewage treatments, polluted and unpolluted water sources, plant roots (Oren 2014). Their existence in an extreme soil environment might be due to their properties of being able to utilize a range of carbon sources like nitrate, oxygen, selenate, chlorate, and perchlorate (Oren 2014). They are primarily anoxygenic photoheterotrophs, and can even function as nitrogen-fixing aerobes, sulfur-oxidizing, methylotrophic, and propionic-acid fermentation (Oren 2014). Vulgatibacteraceae has been previously isolated from forest soils (Yamamoto, Muramatsu, and Nagai 2014), and Woeseiaceae from coastal sediments (Coskun et al. 2019). Woeseiaceae are carbon-fixing microbes which oxidizes compounds with reduced sulfur in extreme coastal environments (Coskun et al. 2019).

However, associating direct functional mechanisms to such a diverse group(s) can be misleading as there are so many species and strains within each phylum and family. We are 
motivated to conduct further functional analyses at the species level, in order to elucidate the genomic functions that are essential to bacterial populations during drought conditions.

\subsection{Soil moisture has a significant impact on selected bacterial genomes and function}

We used post-hoc SIMPER analyses to detect the contribution to dissimilarity at the species level between the dry and wet soil, and used that information to select fifteen bacterial genomes for further gene level analyses (Figure 4, Supplementary Table: S8).

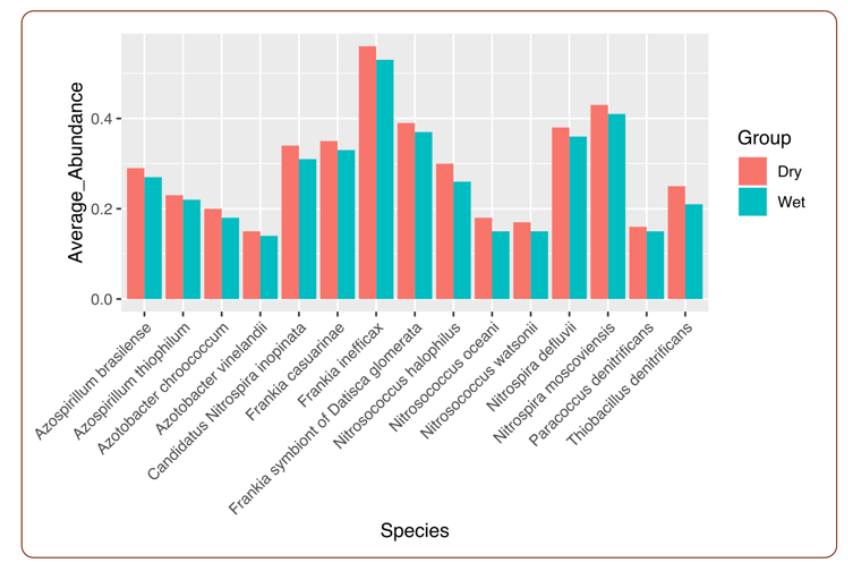

Figure 4

We used Bowtie2 (Langmead and Salzberg 2012) to map the 12 metagenomes to the 15 downloaded bacterial genomes. We performed PERMANOVA statistical analyses, and showed that soil drying had a significant effect on these bacterial populations (PERMANOVA, Pseudo-F 7.2352, $\mathrm{P}<0.05$, Figure 5). We observed that Azotobacter vinelandii (dry: 1.82\%, wet: 1.51\%), Azotobacter chroococcum (dry: 1.96\%, wet: 1.66\%), Nitrosococcus watsonii (dry: 2.04\%, wet: 1.75\%), Nitrosococcus halophilus (dry: 1.8\%, wet: 1.52\%), Nitrosococcus oceani (dry: 1.92\%, wet: 1.66\%), and Thiobacillus denitrificans (dry: 2.84\%, wet: $2.67 \%$ ) had a higher average relative abundance in dry soil than in wet (Supplementary Table: S9). On the other hand, Frankia casuarinae (dry: 3.93\%, wet: 4.34\%), Frankia inefficax (dry: 3.11\%, wet: 3.51\%), Frankia Datisca glomerata (dry: 3.86\%, wet: 4.15\%), showed a higher average relative abundance in wet soil (Supplementary Table: S9). 


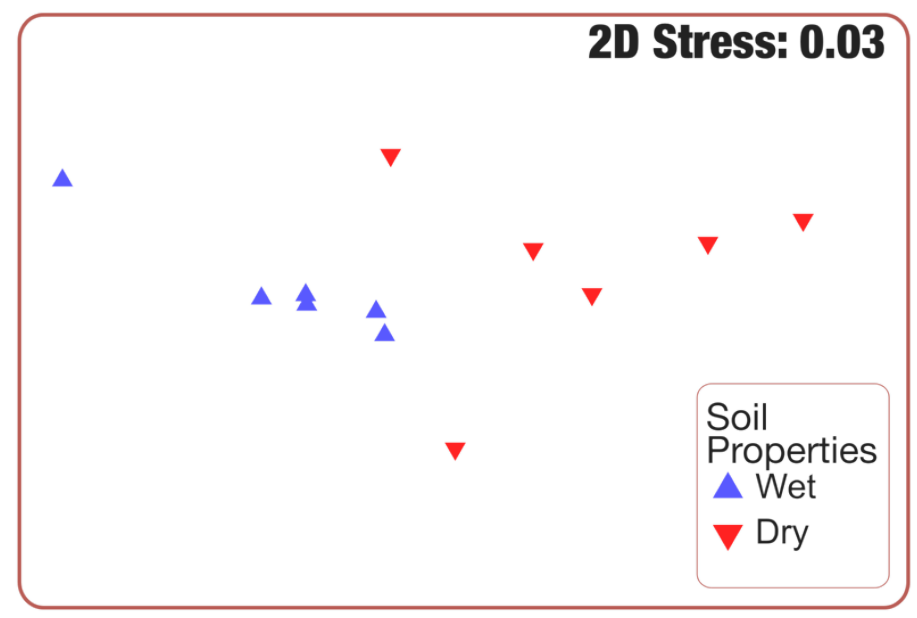

Figure 5

It was evident that the genus Azotobacter, Nitrosococcus, and Thiobacillus were more prevalent in dry conditions, and Frankia was more abundant in wet soil. We believed that being the fact that it is a very well-known biological nitrogen-fixer (Santi, Bogusz, and Franche 2013) was one of the reasons Frankia is present in both wet and dry soil. Interestingly, Azotobacter is capable of surviving in dry soil conditions, resisting tough environmental conditions such as drying or radiations (Vela 1974; Socolofsky and Wyss 1961; Velva and Wyss 1965), by showing some dormant structures, reported to be cysts (Socolofsky and Wyss 1961). These reports corroborate well with our studies that find them more abundant in dry soil. The higher average relative abundance of Thiobacillus denitrificans, Nitrosococcus watsonii, Nitrosococcus halophilus, Nitrosococcus oceani in the dry soil can be potentially interesting as these species are related to various biogeochemical cycles (Prosser 2005; Pajares and Bohannan 2016). Based on these results and subsequent analyses, we hypothesized that these selected bacterial genomes on a whole were influenced by soil moisture content, and that we exploited genomics information within these genomes to gain further insights into their mechanisms during times of drought stress.

We used post-hoc SIMPER analysis on all of the genes in each of the 15 downloaded genomes (Supplementary Table S10). SIMPER test yielded gene lists of the top genes with average relative abundance in both types of soils. Based on that, we selected top 20 genes in most of the 15 downloaded genomes (Supplementary Table S10). Among those genes, we observed a higher coverage of translation elongation factor EF-Tu, thiamine biosynthesis protein, and catalase among the fifteen genomes in which these genes were present in the dry as compared to the wet soil, and 
speculated that these three genes are crucial in enabling bacterial populations to be more resilient during drought and/or high heat conditions (Figure 6).

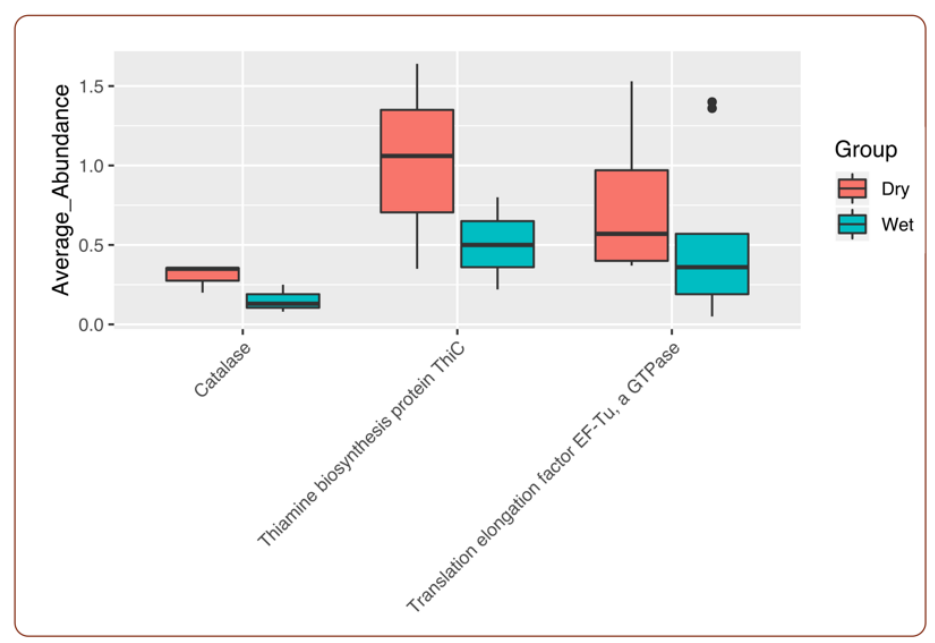

Figure 6

We noticed that translation elongation factor thermo unstable (EF-Tu) was one of the topcontributing genes in Azosprillum brasilense, Azospirillum thiophilum, Azotobacter vinelandii, Candidatus Nitrospira inopinata, Frankia casuarinae, Frankia inefficax, Frankia Datisca glomerata, Nitrospira moscoviensis, and Paracoccus denitrificans (Figure 6, Supplementary Table S11), suggesting that EF-Tu might be one of the mechanisms in which bacterial populations used in overcoming stressful conditions. EF-Tu is one of the most abundant proteins that is found in the bacteria and contributes to almost 6\% of the total expressible protein in Escherichia coli (Furano 1975). Although the primary role of EF-Tu is attaching aminoacyl-tRNA to the ribosome of the cells (Sprinzl 1994), there are other significant diverse roles in various microorganisms. In bacteria, EF-Tu has also been found to protect proteins from stress by forming stable complexes under heat shock conditions, and thus aiding in protein folding and renaturation in the cytoplasm (Caldas, El Yaagoubi, and Richarme 1998) Thus, there have been reports for EF-Tu to protect proteins during heat stress, and assist folding of a number of proteins such as citrate synthase and $\alpha$-glucosidase (Caldas, El Yaagoubi, and Richarme 1998).

We also noticed in our study, thiamine biosynthesis protein was one of the top-most contributing genes in Azotobacter chroococcum, Azotobacter vinelandii, Frankia Datisca glomerata (Figure 6, Supplementary Table S12). Our data suggested that these bacterial 
populations may use thiamine biosynthesis protein to synthesize thiamine, helping these bacterial populations to respond directly to the drought induced oxidative stress by interacting with free radicals (Wolak et al. 2014). In a more indirect approach, synthesized thiamine can also function as a cofactor as transketolase and $\alpha$-ketoglutarate dehydrogenase which influences the redox status of the cells (Bunik 2003; Rapala-Kozik, Kowalska, and Ostrowska 2008). In Escherichia coli, there are reports that thiamine triphosphate (TTP) and its derivative (AThTP) overexpressed under amino acid or carbon stress, acting as 'alarmones' (Lakaye et al. 2004; Gigliobianco et al. 2010).

Similarly, our analysis showed catalase contributed most in Azospirillum brasilense, Frankia casuarinae, and Frankia Datisca glomerata (Figure 6, Supplementary Table S13). We hypothesized that catalase, being a popular antioxidant enzyme known for detoxification of reactive oxygen species (Schellhorn 1995), enabled the bacterial populations to survive stress by degrading molecules such as $\mathrm{H}_{2} \mathrm{O}_{2}$. Catalase is omnipresent in a range of species from bacteria to humans, and neutralizes the toxic $\mathrm{H}_{2} \mathrm{O}_{2}$, converting to oxygen and water (Zamocky, Furtmüller, and Obinger 2008). Currently, there are three classes of catalase, namely: catalase, catalaseperoxidase, and pseudocatalase (X. Liu and Kokare 2017), each class involved in performing the reaction: $\left(2 \mathrm{H}_{2} \mathrm{O}_{2} \rightarrow 2 \mathrm{H}_{2} \mathrm{O}+\mathrm{O}_{2}\right.$ ). We proposed that our short-listed genomes should be divided into three distinct groups and a common mechanism that might be contributing to each group's functioning and resilience to dry conditions (Supplementary Figure 1). In our study, EF-Tu, thiamine biosynthesis, and catalase had more coverage in dry soil than in wet in each of the genomes, and also belong to the top contributing genes in the respective organisms. Our results suggested that bacterial populations may use these mechanisms to enhance their resistance in terms of drought stress.

Our study summarizes the shifts in the microbial structure, as well as in the functions impacted by soil dryness. There were definite impacts of soil dryness on the bacterial community. In dry soil, we demonstrated that there was an increase in the average relative abundance of some families and species, which might have important biological significance(s). Moreover, by shortlisting some important bacterial populations, we explored their potential pathways, and deciphered genes such as EF-Tu, thiamine biosynthesis, and catalase can be key to those microorganisms in aiding their resistance against drought stress. To conclude, in this study, we not only aimed at elucidating the shifts in the bacterial population at the community level and at the genome level but also explore further into the potential mechanisms that the population might use in moisture- 
bioRxiv preprint doi: https://doi.org/10.1101/2020.09.30.320879; this version posted October 1, 2020. The copyright holder for this preprint (which was not certified by peer review) is the author/funder. All rights reserved. No reuse allowed without permission.

deprived environments. This research approach can be applied globally and across different environmental systems, providing insights into deciphering the bacterial community composition and the microbial functional mechanisms. 


\section{Acknowledgments}

Soumyadev Sarkar acknowledges the National Science Foundation EPSCoR for his research grant. Kaitlyn Ward was supported by the research award from the College of Arts and Sciences, Kansas State University.

\section{Conflict of interests}

All authors declare that they have no conflict of interest. 


\section{Figures and Tables legends:}

Figure 1: Microbial composition between wet (soil A) and dry (soil B) soil.

Metagenomic shotgun sequencing data were assigned taxonomic identity using Kaiju and analyzed by Bray-Curtis dissimilarity between the two groups. NMDS plot shows that microbial composition from soil A and B formed distinct clusters.

Figure 2: Key bacterial populations in wet and dry soil.

Taxonomy of bacterial populations assigned using Kaiju showing (A) the relative abundance of

Streptomycetaceae, Bradyrhizobiaceae, Vicinamibacteraceae, Pseudonocardiaceae, and

Planctomycetaceae in dry soil. (B) the relative abundance of Bradyrhizobiaceae,

Streptomycetaceae, Hyphomicrobiaceae, Pseudonocardiaceae, and Vicinamibacteraceae in wet soil.

Figure 3. Top bacterial populations contributors for both dry and wet soil.

Metagenomic shotgun sequencing data was analyzed using PERMANOVA and top contributors were identified using post-hoc SIMPER test.

Figure 4: Distribution of the 15 downloaded key genomes in wet and dry soil.

Relative abundance of the 15 genomes in the dry and wet soil, showing that all the 15 genomes have a higher relative abundance in the dry soil than in wet. Nitrosococcus halophilus and Thiobacillus denitrificans show the highest relative abundance in the dry soil when compared to wet among the 15 genomes that we selected.

Figure 5: Relative abundance of 15 downloaded key genomes in wet and dry soil. Metagenomic shotgun sequencing data were mapped to the 15 downloaded genomes and analyzed by Bray-Curtis dissimilarity between the two groups. NMDS plot shows that the relative abundance of the 15 genomes composition from soil A and B formed distinct clusters. 
Figure 6: Distribution of EF-Tu, thiamine biosynthesis, and catalase gene function in wet and dry soil. EF-Tu, thiamine biosynthesis, and catalase within the 15 genomes have significantly higher relative abundance in dry as compared to wet soil. These three bacterial genes are associated with microbial resilience during drought conditions.

Supplementary Table 1: Details of the 15 downloaded genomes.

Supplementary Table 2: Assembly statistics.

Supplementary Table 3: Classification of 365 families in both the soil locations.

Supplementary Table 4: Families that were top contributors in the dry soil.

Supplementary Table 5: Families that were top contributors in the wet soil.

Supplementary Table 6: SIMPER analysis indicated the top contributors in dry soil when compared to wet.

Supplementary Table 7: SIMPER analysis detected $~ 110$ families that were overlapping in terms of average relative abundance in both wet and dry soil. Families that were exclusive to the dry and the wet are indicated with red colored font.

Supplementary Table 8: Relative abundance of 15 downloaded genomes in dry and wet soil.

Supplementary Table 9: Bacterial population among 15 genomes that show difference in relative abundance and also belong to the top contributors. 
Supplementary Table 10: SIMPER analysis on all of the genes in each of the 15 downloaded genomes and $\sim$ top 20 genes in most of the 15 downloaded genomes.

Supplementary Table 11: Distribution of EF-Tu gene function in wet and dry soil.

Supplementary Table 12: Distribution of thiamine biosynthesis gene function in wet and dry soil.

Supplementary Table 13: Distribution catalase gene function in wet and dry soil.

Supplementary Figure 1: Individual pathways for the genomes. 


\section{References}

Avolio, Meghan L., Jeremy M. Beaulieu, and Melinda D. Smith. 2013. "Genetic Diversity of a Dominant C4 Grass Is Altered with Increased Precipitation Variability." Oecologia 171 (2): 571-81.

Barnard, Romain L., Catherine A. Osborne, and Mary K. Firestone. 2013. "Responses of Soil Bacterial and Fungal Communities to Extreme Desiccation and Rewetting." The ISME Journal 7 (11): 2229-41.

Bouskill, Nicholas J., Tana E. Wood, Richard Baran, Zaw Ye, Benjamin P. Bowen, Hsiaochien Lim, Jizhong Zhou, et al. 2016. "Belowground Response to Drought in a Tropical Forest Soil. I. Changes in Microbial Functional Potential and Metabolism." Frontiers in Microbiology 7 (April): 525.

Bruce, Thiago, Ivana B. Martinez, Oswaldo Maia Neto, Ana Carolina P. Vicente, Ricardo H. Kruger, and Fabiano L. Thompson. 2010. "Bacterial Community Diversity in the Brazilian Atlantic Forest Soils.” Microbial Ecology. https://doi.org/10.1007/s00248-010-9750-2.

Buckley, Daniel H., Varisa Huangyutitham, Tyrrell A. Nelson, Angelika Rumberger, and Janice E. Thies. 2006. "Diversity of Planctomycetes in Soil in Relation to Soil History and Environmental Heterogeneity." Applied and Environmental Microbiology 72 (7): 4522-31.

Buckley, D. H., and T. M. Schmidt. 2001. "Environmental Factors Influencing the Distribution of rRNA from Verrucomicrobia in Soil." FEMS Microbiology Ecology 35 (1): 105-12.

Bunik, Victoria I. 2003. "2-Oxo Acid Dehydrogenase Complexes in Redox Regulation. Role of the Lipoate Residues and Thioredoxin.” European Journal of Biochemistry. https://doi.org/10.1046/j.1432-1033.2003.03470.x.

Caldas, Teresa Dantas, Abdelhamid El Yaagoubi, and Gilbert Richarme. 1998. "Chaperone Properties of Bacterial Elongation Factor EF-Tu." Journal of Biological Chemistry. https://doi.org/10.1074/jbc.273.19.11478.

Chang, Woo-Suk, Martijn van de Mortel, Lindsey Nielsen, Gabriela Nino de Guzman, Xiaohong Li, and Larry J. Halverson. 2007. "Alginate Production by Pseudomonas Putida Creates a Hydrated Microenvironment and Contributes to Biofilm Architecture and Stress Tolerance under Water-Limiting Conditions." Journal of Bacteriology. https://doi.org/10.1128/jb.00727-07.

Chikere, Chioma Blaise, Ijeoma Jessie Mordi, Blaise Ositadinma Chikere, Ramganesh Selvarajan, Tom Omotayo Ashafa, and Chinedu Christopher Obieze. 2019. "Comparative Metagenomics and Functional Profiling of Crude Oil-Polluted Soils in Bodo West Community, Ogoni, with Other Sites of Varying Pollution History." Annals of Microbiology 69 (5): 495-513.

Chodak, Marcin, Marcin Gołębiewski, Justyna Morawska-Płoskonka, Katarzyna Kuduk, and Maria Niklińska. 2015. "Soil Chemical Properties Affect the Reaction of Forest Soil Bacteria to Drought and Rewetting Stress." Annals of Microbiology 65 (3): 1627-37.

Chowdhury, Taniya Roy, Joon-Yong Lee, Eric M. Bottos, Colin J. Brislawn, Richard Allen White, Lisa M. Bramer, Joseph Brown, et al. 2019. "Metaphenomic Responses of a Native Prairie Soil Microbiome to Moisture Perturbations." mSystems. https://doi.org/10.1128/msystems.00061-19.

Christian, Keith, Mirjam Kaestli, and Karen Gibb. 2017. "Spatial Patterns of Hypolithic Cyanobacterial Diversity in Northern Australia." Ecology and Evolution 7 (17): 7023-33. 
Clarke, K. R., and R. N. Gorley. 2015. "PRIMER v7: User Manual/tutorial 3rd Ed.” Plymouth, United Kingdom: Primer-E Ltd.

Cook, Benjamin I., Richard Seager, and Ron L. Miller. 2011. "Atmospheric Circulation Anomalies during Two Persistent North American Droughts: 1932-1939 and 1948-1957." Climate Dynamics 36 (11): 2339-55.

Coskun, Ömer K., Volkan Özen, Scott D. Wankel, and William D. Orsi. 2019. "Quantifying Population-Specific Growth in Benthic Bacterial Communities under Low Oxygen Using H218O." The ISME Journal. https://doi.org/10.1038/s41396-019-0373-4.

Eren, A. M., Ö. C. Esen, C. Quince, J. H. Vineis, H. G. Morrison, and M. L. Sogin. 2015. “Anvi'o: An Advanced Analysis and Visualization Platform for 'omics Data." PeerJ 3. https://doi.org/10.7717/peerj.1319.

Fierer, Noah, Mark A. Bradford, and Robert B. Jackson. 2007. "Toward an Ecological Classification of Soil Bacteria.” Ecology 88 (6): 1354-64.

Furano, A. V. 1975. "Content of Elongation Factor Tu in Escherichia Coli." Proceedings of the National Academy of Sciences. https://doi.org/10.1073/pnas.72.12.4780.

Gigliobianco, Tiziana, Bernard Lakaye, Pierre Wins, Benaïssa El Moualij, Willy Zorzi, and Lucien Bettendorff. 2010. "Adenosine Thiamine Triphosphate Accumulates in Escherichia Coli Cells in Response to Specific Conditions of Metabolic Stress.” BMC Microbiology 10 (May): 148.

Gómez-Rubio, Virgilio. 2017. "ggplot2 - Elegant Graphics for Data Analysis (2nd Edition)." Journal of Statistical Software. https://doi.org/10.18637/jss.v077.b02.

Gornall, Jemma, Richard Betts, Eleanor Burke, Robin Clark, Joanne Camp, Kate Willett, and Andrew Wiltshire. 2010. "Implications of Climate Change for Agricultural Productivity in the Early Twenty-First Century." Philosophical Transactions of the Royal Society of London. Series B, Biological Sciences 365 (1554): 2973-89.

Goto, Keiichi, Kaoru Mochida, Mika Asahara, Masayuki Suzuki, Hiroaki Kasai, and Akira Yokota. 2003. “Alicyclobacillus Pomorum Sp. Nov., a Novel Thermo-Acidophilic, Endospore-Forming Bacterium That Does Not Possess $\omega$-Alicyclic Fatty Acids, and Emended Description of the Genus Alicyclobacillus." International Journal of Systematic and Evolutionary Microbiology. https://doi.org/10.1099/ijs.0.02546-0.

Gupta, Abhishek, Avishek Dutta, Jayeeta Sarkar, Mruganka Kumar Panigrahi, and Pinaki Sar. 2018. "Low-Abundance Members of the Firmicutes Facilitate Bioremediation of Soil Impacted by Highly Acidic Mine Drainage From the Malanjkhand Copper Project, India." Frontiers in Microbiology. https://doi.org/10.3389/fmicb.2018.02882.

Heisler-White, Jana L., John M. Blair, Eugene F. Kelly, Keith Harmoney, and Alan K. Knapp. 2009. "Contingent Productivity Responses to More Extreme Rainfall Regimes across a Grassland Biome.” Global Change Biology 15 (12): 2894-2904.

Imperio, Tatiana, Carlo Viti, and Laura Marri. 2008. “Alicyclobacillus Pohliae Sp. Nov., a Thermophilic, Endospore-Forming Bacterium Isolated from Geothermal Soil of the NorthWest Slope of Mount Melbourne (Antarctica)." International Journal of Systematic and Evolutionary Microbiology 58 (Pt 1): 221-25.

Intergovernmental Panel on Climate Change. 2014. Climate Change 2013 - The Physical Science Basis: Working Group I Contribution to the Fifth Assessment Report of the Intergovernmental Panel on Climate Change. Cambridge University Press.

Jones, Sydney K., Scott L. Collins, John M. Blair, Melinda D. Smith, and Alan K. Knapp. 2016. "Altered Rainfall Patterns Increase Forb Abundance and Richness in Native Tallgrass 
Prairie." Scientific Reports 6 (February): 20120.

Kielak, Anna, Agata S. Pijl, Johannes A. van Veen, and George A. Kowalchuk. 2008.

"Differences in Vegetation Composition and Plant Species Identity Lead to Only Minor

Changes in Soil-Borne Microbial Communities in a Former Arable Field." FEMS

Microbiology Ecology 63 (3): 372-82.

Knapp, Alan K., Philip A. Fay, John M. Blair, Scott L. Collins, Melinda D. Smith, Jonathan D.

Carlisle, Christopher W. Harper, Brett T. Danner, Michelle S. Lett, and James K.

McCarron. 2002. "Rainfall Variability, Carbon Cycling, and Plant Species Diversity in a

Mesic Grassland." Science 298 (5601): 2202-5.

Kuang, Shaoping, Yaqing Su, Huihui Wang, Wenjuan Yu, Qiaolin Lang, and Ravichandra

Matangi. 2018. "Soil Microbial Community Structure and Diversity around the Aging Oil

Sludge in Yellow River Delta as Determined by High-Throughput Sequencing." Archaea 2018 (August): 7861805.

Lakaye, Bernard, Barbara Wirtzfeld, Pierre Wins, Thierry Grisar, and Lucien Bettendorff. 2004.

"Thiamine Triphosphate, a New Signal Required for Optimal Growth of Escherichia Coli

during Amino Acid Starvation." The Journal of Biological Chemistry 279 (17): 17142-47.

Langmead, Ben, and Steven L. Salzberg. 2012. "Fast Gapped-Read Alignment with Bowtie 2."

Nature Methods 9 (4): 357-59.

Lau, Jennifer A., and Jay T. Lennon. 2012. "Rapid Responses of Soil Microorganisms Improve

Plant Fitness in Novel Environments." Proceedings of the National Academy of Sciences of

the United States of America 109 (35): 14058-62.

Li, Heng, Bob Handsaker, Alec Wysoker, Tim Fennell, Jue Ruan, Nils Homer, Gabor Marth,

Goncalo Abecasis, Richard Durbin, and 1000 Genome Project Data Processing Subgroup.

2009. "The Sequence Alignment/Map Format and SAMtools." Bioinformatics 25 (16):

2078-79.

Liu, Dong, Katharina M. Keiblinger, Sonja Leitner, Uwe Wegner, Michael Zimmermann,

Stephan Fuchs, Christian Lassek, Katharina Riedel, and Sophie Zechmeister-Boltenstern.

2019. "Response of Microbial Communities and Their Metabolic Functions to Drying-

Rewetting Stress in a Temperate Forest Soil." Microorganisms 7 (5): 129.

Liu, Xiangyang, and Chandrakant Kokare. 2017. "Chapter 11 - Microbial Enzymes of Use in

Industry." In Biotechnology of Microbial Enzymes, edited by Goutam Brahmachari, 267-

98. Academic Press.

Marasco, Ramona, Eleonora Rolli, Besma Ettoumi, Gianpiero Vigani, Francesca Mapelli, Sara

Borin, Ayman F. Abou-Hadid, et al. 2012. "A Drought Resistance-Promoting Microbiome

Is Selected by Root System under Desert Farming." PLoS ONE.

https://doi.org/10.1371/journal.pone.0048479.

Marín, I., and D. Ruiz Arahal. 2014. “The Family Beijerinckiaceae.” The Prokaryotes, 115-33.

Menzel, Peter, Kim Lee Ng, and Anders Krogh. 2016. "Fast and Sensitive Taxonomic

Classification for Metagenomics with Kaiju." Nature Communications.

https://doi.org/10.1038/ncomms11257.

Miyasaka, Natália Reiko Sato, Daniela Strauss Thuler, Eny Iochevet Segal Floh, Walter Handro,

Mariana Braga Duarte Toledo, Sônia Maria Gagioti, and Heloiza Ramos Barbosa. 2003.

"During Stationary Phase, Beijerinckia Derxii Shows Nitrogenase Activity Concomitant

with the Release and Accumulation of Nitrogenated Substances." Microbiological

Research 158 (4): 309-15.

Navarrete, Acacio Aparecido, Tatiana Rosa Diniz, Lucas Palma Perez Braga, Genivaldo Gueiros 
Zacarias Silva, Julio Cezar Franchini, Raffaella Rossetto, Robert Alan Edwards, and Siu Mui Tsai. 2015. "Multi-Analytical Approach Reveals Potential Microbial Indicators in Soil for Sugarcane Model Systems." PloS One 10 (6): e0129765.

Navarrete, Acacio Aparecido, Tielle Soares, Raffaella Rossetto, Johannes Antonie van Veen, Siu Mui Tsai, and Eiko Eurya Kuramae. 2015. "Verrucomicrobial Community Structure and Abundance as Indicators for Changes in Chemical Factors Linked to Soil Fertility." Antonie van Leeuwenhoek 108 (3): 741-52.

Navarrete, Acacio A., Siu M. Tsai, Lucas W. Mendes, Karoline Faust, Mattias de Hollander, Noriko A. Cassman, Jeroen Raes, Johannes A. van Veen, and Eiko E. Kuramae. 2015. "Soil Microbiome Responses to the Short-Term Effects of Amazonian Deforestation." Molecular Ecology 24 (10): 2433-48.

Oren, Aharon. 2014. "The Family Rhodocyclaceae.” In The Prokaryotes: Alphaproteobacteria and Betaproteobacteria, edited by Eugene Rosenberg, Edward F. DeLong, Stephen Lory, Erko Stackebrandt, and Fabiano Thompson, 975-98. Berlin, Heidelberg: Springer Berlin Heidelberg.

Pajares, Silvia, and Brendan J. M. Bohannan. 2016. "Ecology of Nitrogen Fixing, Nitrifying, and Denitrifying Microorganisms in Tropical Forest Soils." Frontiers in Microbiology 7 (July): 1045.

Pajares, Silvia, Julio Campo, Brendan J. M. Bohannan, and Jorge D. Etchevers. 2018. "Environmental Controls on Soil Microbial Communities in a Seasonally Dry Tropical Forest." Applied and Environmental Microbiology 84 (17). https://doi.org/10.1128/AEM.00342-18.

Pan, Yao, Noriko Cassman, Mattias de Hollander, Lucas W. Mendes, Hein Korevaar, Rob H E, Johannes A. van Veen, and Eiko E. Kuramae. 2014. "Impact of Long-Term N, P, K, and NPK Fertilization on the Composition and Potential Functions of the Bacterial Community in Grassland Soil.” FEMS Microbiology Ecology. https://doi.org/10.1111/15746941.12384.

Prosser, J. I. 2005. "NITROGEN IN SOILS | Nitrification.” Encyclopedia of Soils in the Environment. https://doi.org/10.1016/b0-12-348530-4/00512-9.

Rapala-Kozik, Maria, Ewa Kowalska, and Katarzyna Ostrowska. 2008. "Modulation of Thiamine Metabolism in Zea Mays Seedlings under Conditions of Abiotic Stress." Journal of Experimental Botany 59 (15): 4133-43.

Riah-Anglet, Wassila, Isabelle Trinsoutrot-Gattin, Fabrice Martin-Laurent, Emilie LarocheAjzenberg, Marie-Paule Norini, Xavier Latour, and Karine Laval. 2015. "Soil Microbial Community Structure and Function Relationships: A Heat Stress Experiment." Applied Soil Ecology: A Section of Agriculture, Ecosystems \& Environment 86 (February): 121-30.

Roberson, E. B., and M. K. Firestone. 1992. "Relationship between Desiccation and Exopolysaccharide Production in a Soil Pseudomonas Sp." Applied and Environmental Microbiology 58 (4): 1284-91.

Santi, Carole, Didier Bogusz, and Claudine Franche. 2013. "Biological Nitrogen Fixation in Non-Legume Plants." Annals of Botany 111 (5): 743-67.

Sayers, Eric W., Jeff Beck, J. Rodney Brister, Evan E. Bolton, Kathi Canese, Donald C. Comeau, Kathryn Funk, et al. 2020. "Database Resources of the National Center for Biotechnology Information." Nucleic Acids Research 48 (D1): D9-16.

Schellhorn, H. E. 1995. "Regulation of Hydroperoxidase (catalase) Expression in Escherichia Coli." FEMS Microbiology Letters 131 (2): 113-19. 
Schimel, Joshua P. 2018. "Life in Dry Soils: Effects of Drought on Soil Microbial Communities and Processes." Annual Review of Ecology, Evolution, and Systematics 49 (1): 409-32.

Selim, Samy, Yasser M. Hassan, Ahmed M. Saleh, Talaat H. Habeeb, and Hamada AbdElgawad. 2019. "Actinobacterium Isolated from a Semi-Arid Environment Improves the Drought Tolerance in Maize (Zea Mays L.)." Plant Physiology and Biochemistry: PPB / Societe Francaise de Physiologie Vegetale 142 (September): 15-21.

Socolofsky, M. D., and O. Wyss. 1961. "CYSTS OF AZOTOBACTER.” Journal of Bacteriology 81 (6): 946-54.

Sprinzl, M. 1994. "Elongation Factor Tu: A Regulatory GTPase with an Integrated Effector." Trends in Biochemical Sciences 19 (6): 245-50.

Stackebrandt, E. 2014. "The Order Glycomycetales and the Genus Actinocatenispora." The Prokaryotes, Eds E. Rosenberg, EF DeLong, S. Lory, E. Stackebrandt, and F. Thompson (Berlin: Springer), 381-90.

Taylor, Christopher M., Richard A. M. de Jeu, Françoise Guichard, Phil P. Harris, and Wouter A. Dorigo. 2012. "Afternoon Rain More Likely over Drier Soils." Nature 489 (7416): 42326.

Thuler, D. S., E. I. S. Floh, W. Handro, and H. R. Barbosa. 2003. "Beijerinckia Derxii Releases Plant Growth Regulators and Amino Acids in Synthetic Media Independent of Nitrogenase Activity." Journal of Applied Microbiology 95 (4): 799-806.

Vela, G. R. 1974. "Survival of Azotobacter in Dry Soil." Applied Microbiology 28 (1): 77-79.

Velva, G. R., and O. Wyss. 1965. "RADIATION RESISTANCE OF SOIL AZOTOBACTER." Journal of Bacteriology 89 (May): 1280-85.

VijayaVenkataRaman, S., S. Iniyan, and Ranko Goic. 2012. "A Review of Climate Change, Mitigation and Adaptation." Renewable and Sustainable Energy Reviews 16 (1): 878-97.

Ward, Naomi L., Jean F. Challacombe, Peter H. Janssen, Bernard Henrissat, Pedro M. Coutinho, Martin Wu, Gary Xie, et al. 2009. "Three Genomes from the Phylum Acidobacteria Provide Insight into the Lifestyles of These Microorganisms in Soils." Applied and Environmental Microbiology 75 (7): 2046-56.

Wolak, Natalia, Ewa Kowalska, Andrzej Kozik, and Maria Rapala-Kozik. 2014. "Thiamine Increases the Resistance of Baker's yeast Saccharomyces Cerevisiae against Oxidative, Osmotic and Thermal Stress, through Mechanisms Partly Independent of Thiamine Diphosphate-Bound Enzymes." FEMS Yeast Research. https://doi.org/10.1111/15671364.12218.

$\mathrm{Xu}$, Ling, and Devin Coleman-Derr. 2019. "Causes and Consequences of a Conserved Bacterial Root Microbiome Response to Drought Stress.” Current Opinion in Microbiology 49 (June): 1-6.

Yamamoto, Eisaku, Hideyuki Muramatsu, and Koji Nagai. 2014. "Vulgatibacter Incomptus Gen. Nov., Sp. Nov. and Labilithrix Luteola Gen. Nov., Sp. Nov., Two Myxobacteria Isolated from Soil in Yakushima Island, and the Description of Vulgatibacteraceae Fam. Nov., Labilitrichaceae Fam. Nov. and Anaeromyxobacteraceae Fam. Nov." International Journal of Systematic and Evolutionary Microbiology. https://doi.org/10.1099/ijs.0.063198-0.

Zamocky, Marcel, Paul G. Furtmüller, and Christian Obinger. 2008. "Evolution of Catalases from Bacteria to Humans." Antioxidants \& Redox Signaling 10 (9): 1527-48.

Zhou, Xue, Dario Fornara, Makoto Ikenaga, Isao Akagi, Ruifu Zhang, and Zhongjun Jia. 2016. "The Resilience of Microbial Community under Drying and Rewetting Cycles of Three Forest Soils.” Frontiers in Microbiology 7 (July): 1101. 